\title{
Deutsche Gentechnik-Gesetzgebung
}

\author{
Dr.-Ing. U.-D. MATZKE \\ Ministerium für Umwelt, Raumordnung und Landwirtschaft (MURL) NRW, Schwannstraße 3, D-4000 Düsseldorf 1
}

In verschiedenen Gesetzen und Rechtsverordnungen verstreute gentechnikrechtliche Bestimmungen und die seit Asilomar in Anlehnung an die NH-Guidelines fortgeschriebenen, nur für die geförderte Forschung verbindlichen „Richtlinien zum Schutz vor Gefahren durch in-vitro neukombinierte Nukleinsäuren - Genrichtlinien des BMFT“, erforderten einen legislativen Regelungsbedarf. Diesen hatte auch die Enquête-Kommission des Deutschen Bundestages „Chancen und Risiken der Gentechnologie" vorgeschlagen. Bedingt durch den rechtlich nicht haltbaren Beschluß des VGH Kassel vom 06. 11. 1989 wurde das Gesetzgebungsverfahren stark beschleunigt, wobei das zeitgleich beratene europäische Recht in Form von EG-Richtlinien schon miteinbezogen werden konnte.

Das Gentechnik-Recht ist eine neue Rechtsmaterie, die $\mathrm{Na}$ turwissenschaft und Recht, Forschung und industrielle Nutzung in einem Normenwerk zusammenbringt. Es will vor allem Leben, Gesundheit und Umwelt durch ein differenziert zu handhabendes System des Verbots mit Erlaubnisvorbehalt schützen und dem Entstehen von Gefahren aus gentechnischen Anlagen vorbeugen.

Das neue Recht ergab eine Reihe von Umsetzungsschwierigkeiten bei den Betreibern gentechnischer Anlagen sowie bei den Behörden, ausgelöst durch Zweifelsfragen bei der Auslegung des Gesetzestextes und den im GentechnikGesetz eingearbeiteten Verschärfungen gegenüber dem EGRecht.

Diese Verschärfungen, die Einführung eines formularisierten Anmelde- und Genehmigungsverfahrens, eine von der Wissenschaft zum Teil falsch verstandene Forschungsfreiheit und die fehlende Akzeptanz der Gentechnik in Deutschland werden gegenüber dem gesetzlich verbrieften Rechtsanspruch auf Nutzung der Gentechnik als zum Teil unüberwindbare Hemmnisse angesehen.

1. In der Diskussion um die Anwendung der Gentechnik spielt der Begriff des Risikos eine entscheidende Rolle. Die Abwägung zwischen Risiko und Nutzen bei der Anwendung der Gentechnik ist wegen der hohen Komplexität auBerordentlich schwer. Eine Risikobetrachtung der Gentechnik als solche i.S. des technischen Risikobegriffs ist nicht möglich, da sie zunächst nur das Handwerkszeug beschreibt, das Erbgut von Lebewesen zu verändern. Wie hoch das Risiko durch solche Änderungen ist, läßt sich wenn überhaupt - nur im konkreten Fall beantworten, zumal die Produkte der Gentechnik auch Lebenwesen sein können, die unter bestimmten Umständen in der Umwelt vermehrungsfähig und dann evtl. nicht mehr rückholbar sind. Insoweit bietet auch die Schweizer Störfallverordnung (StFV) keine neuen Ansatzpunkte und Betrachungsweisen. Sicherheit ist nach meiner Überzeugung unteilbar. Sicherheit ist auch nicht durch eine Behörde in eine Anlage hineinzuprüfen, sondern sie muß wegen der Frage der Rückhol- barkeit durch die Ausführung der Arbeit und der Anlage vom Betreiber gewährleistet werden.

Dieses auf beiden Seiten erforderliche neue Sicherheitsverständnis weitet die bislang betrachteten Bereiche des Schutzes der Arbeitnehmer und der Nachbarschaft erheblich aus, da umfangreiche Kenntnisse über die Eigenschaften von (gentechnisch veränderten) Organismen und ihren Wechselwirkungen mit dem Ökosystem Voraussetzung sind.

2. "Weniger Bürokratie, mehr Deregulierung“ lauten die Schlagworte, die auf die junge und zum Teil noch unerfahrene Verwaltung in Sachen Gentechnik zukommen.

Sofern unter Deregulierung die Aufhebung, Regelungsentfernung, Vereinfachung, Konzentration und Beschleunigung i.V. mit gesetzlichen Bestimmungen verstanden wird, gleicht die Bewältigung dieser Aufgabe der einer nicht enden wollenden Sisyphusarbeit.

Soweit nur die die Gesetze umsetzende Verwaltung gemeint ist, gibt es Ansatzpunkte, die trotz der unumstrittenen Komplexität und Kompliziertheit unserer Rechts- und Gesellschaftsordnung durch kooperative Handlungsformen Erfolge zeitigen können. Als Beispiel dafür seien die Beschlüsse des Länderausschusses für Gentechnik (LAG) veröffentlicht im Deutschen Verwaltungsblatt (DVBl. 1992, S. 1079) - genannt sowie die Überarbeitung und drastische Kürzung der bundeseinheitlich auszufüllenden Formblätter bei der Beantragung gentechnischer Arbeiten, um eine rasche Prüfung der Antragsunterlagen auf Vollständigkeit zu ermöglichen.

Die Staatsverdrossenheit, das Anschwellen der Gesetzestexte und ihre zunehmende Unübersichtlichkeit und zum Teil innere Widersprüchlichkeit nehmen rapide zu und nicht $a b$. Das noch junge Pflänzchen Gentechnik fühlt sich nicht nur durch die gesetzlichen, sondern auch durch die verwaltungsmäßigen Vorgaben im wahrsten Sinne des Wortes vergewaltigt.

3. Der seit Anfang 1992 einsetzende Druck von der Bürokratie sich geknechtet vorkommender Professoren und der Dringlichkeitsappell der Max-Planck-Gesellschaft haben dazu geführt, daß trotz der komplexen Ursachen (gesellschaftliche, politische und gesetzgeberische Vorgaben, ungünstiges Investitionsklima in Verbindung mit Personalkosten, Steuern, Sozialleistungen, Kapitalbelastungen; keine wirtschaftlichen Erfolge signalisierende Forschungsergebnisse) eine Novellierung des Gesetzes initiiert wurde. $\mathrm{Ob}$ sich die Beschlußempfehlungen des Ausschusses für Forschung, Technologie und Technikabfolgenabschätzung des Deutschen Bundestages ohne gleichzeitige Änderung der EG-Richtlinien umsetzen lassen, muß beweifelt werden:

- Abschaffung der Anmeldepflicht zugunsten einer Anzeigepflicht in der Sicherheitsstufe 1 vor dem erstmaligen Beginn von Arbeiten zu Forschungszwecken, 
- Ersatz der Genehmigungspflicht durch ein Anmeldeverfahren für Anlagen und Arbeiten zu Forschungszwecken in der Sicherheitsstufe 2,

- Klarstellung des Begriffs ,kleiner Maßstab“,

- Sicherung eines ungestörten internationalen Austausches von gentechnisch veränderten Organismen zu Forschungszwecken,

- Vereinfachung der Aufzeichnungspflichten und Gebührenbefreiung für gemeinnützige Forschungseinrichtungen.

4. Viel positiver zu werten ist das Einstehen der politisch Verantwortung Tragenden für die Gentechnik in Verbindung mit einer klaren Distanzierung von den Ängsten der Menschen wie Änderung der Keimzellen, Beeinflussung des Geschlechts und der Erbinformation bis hin zur Abschaffung von Erbkrankheiten durch Geburtenkontrolle.

Die Vertrautheit der Bevölkerung mit der Gentechnik und deren positiven Möglichkeiten muß anwachsen und bereits im Lehrstoff der Schulen verankert werden. Es gilt, gemeinfaßliche Erklärungsformen für die Molekulargenetik zu finden und dic Bringschuld der Wissenschaft mit der Holschuld der Journalisten so zu kombinieren, daß die Wissenschaft den Graben erkennt, den die Bevölkerung nicht überschritten wissen möchte.

Forschungsfreiheit in den vom Gentechnik-Gesetz gezogenen Grenzen engt nicht die Forschung ein, sondern bedeutet, daß die Gentechnik von der gesellschaftlichen Diskussion begleitet und in sie eingebunden wird. Wissenschaft ist keine emotionslose Angelegenheit. Neben Fakten müssen auch Meinungen diskutiert werden, so daß eine kritische Auseinandersetzung mit den Argumenten des Gegners möglich ist und die Forschungsfreiheit in Verbindung mit der Eigenverantwortlichkeit des Forschers nicht alleiniges Alibi wird.

\section{Kurznachrichten}

\section{Altpapierverordnung \\ - Entwurf}

Im Rahmen des abfallwirtschaftlichen Gesamtkonzepts von Bundesumweltminister KLaus TÖPFER wurde am 21. 09. 1992 der Entwurf seiner Verordnung über die Vermeidung und Verwertung von Abfällen aus Druckerzeugnissen sowie aus Büro- und Administrationspapieren den beteiligten Wirtschaftskreisen, den Ländern und den Ressorts zugeleitet.

Bei einem Verbrauch von 6,4 Mio. Tonnen belief sich das Abfallaufkommen im Bereich der Druck- und Pressepapiere sowie der Büro- und Administrationspapiere (graphische Papiere) 1990 allein in den alten Bundesländern auf etwa 2,5 Mio. t. Diese Abfälle wurden zu etwa $30 \%$ in Müllverbrennungsanlagen und zu $70 \%$ auf Deponien entsorgt. Das Abfallaufkommen im Bereich der graphischen Papiere überstieg damit sogar das Abfallaufkommen an Verpackungspapieren. Dort fielen 1990 etwa 2,1 Mio. t Abfälle an.

Zwar gelang es mit Hilfe der Altpapierverwertung 3,2 Mio. t gebrauchter graphischer Papiere von Müllverbrennungsanlagen und Deponien fernzuhalten, doch ist dies für die Zukunft nicht ausreichend.

1. Hauptziele der Altpapierverordnung:

- Sicherstellung einer höchstmöglichen stofflichen Verwertung gebrauchter Papiere,

- konsequente Ausschöpfung vorhandener Altpapiereinsatzmöglichkeiten im Rahmen der Neupapiererzeugung,

- technologische Weiterentwicklung der Altpapiereinsatzmöglichkeiten im Rahmen der Papiererzeugung,

- Erschließung von Einsatzmöglichkeiten für Altpapiere außerhalb der Papiererzeugung.

2. Zugleich soll mit der Altpapierverordnung eine Entschließung des Bundesrates vom 19. April 1991 umgesetzt werden. Der Bundesrat hatte damals darauf gedrängt, daß der Bundesumweltminister in Ergänzung zur Verpackungsverordnung eine Rücknahme- und Verwertungsverordnung für Zeitungen, Zeitschriften und sonstige Druckwerke aus Papier vorlegt.

Im einzelnen sieht die Rücknahmeverordnung folgende Regelungen vor:

- Ab 01. Juli 1993 müssen Hersteller und Vertreiber Büro- und Administrationspapiere sowie Druckerzeugnisse nach Gebrauch unentgeltlich vom Verbraucher zurücknehmen. Die Rücknahmepflicht für Druckerzeugnisse gilt dabei insbesondere für Zeitungen, Zeitschriften, Anzeigenblätter und Offertenblätter. Sie erstreckt sich aber auch auf jede Art von verteilter Werbung (Prospekte, Kataloge Handzettel oder Postwurfsendungen).

- Die Rücknahme hat grundsätzlich in unmittelbarer Nähe der Abgabstelle zu erfolgen. Werden Druckerzeugnisse dem Endverbraucher zugestellt oder in sonstiger Weise überlassen, muß der Vertreiber dafür Sorge tragen, daß geeignete unentgeltliche Rückgabe- oder wahlweie Rücksendemöglichkeiten bestehen. Hierauf ist im Druckerzeugnis hinzuweisen, damit sich der Verbraucher darauf einstellen kann.

- Hersteller und Vertreiber sind verpflichtet, die vom Endverbraucher zurückgenommenen graphischen Papiere, aber auch die nicht abgesetzten Druckerzeugnisse, die sog. Remittenden, einer weitestgehenden stofflichen Verwertung außerhalb der öffentlichen Abfallentsorgung zuzuführen.
3. Die Altpapierverordnung schreibt im Hinblick auf die Zulassung dualer Systeme vor:

- Die Erfassung gebrauchter graphischer Papiere beim Endverbraucher kann sowohl über Holsysteme (beispielsweise Bündelsammlung oder Monotonne) als auch über Bringsysteme (z.B. Altpapiercontainer) erfolgen. In jedem Fall ist sicherzustellen, daß die Erfassung flächendeckend und in einer ausreichenden Häufigkeit sowie unentgeltlich für den Endverbraucher erfolgt.

- Vorhandene Sammel- und Sortiereinrichtungen der entsorgungspflichtigen Körperschaften sind vorrangig zu übernehmen. Die Kommunen können die Übernahme ihrer Einrichtungen gegen ein angemessenes Entgelt verlangen, soweit dies die stoffliche Verwertung der erfaßten graphischen Papiere fördert und nicht zu unzumutbaren Mehrkosten gegenüber den geplanten dualen Systemen führt.

- Die Recyclingquote, gemessen am gesamten Verbrauch graphischer Papiere, ist in den kommenden Jahren von gegenwärtig etwa $50 \%$ wie folgt zu steigern:

O bis zum 31. Dezember $1994 \quad 52 \%$ $O$ in der Zeit vom 01. Januar 1995

bis zum 31. Dezember $199655 \%$ O ab 01. Januar $1997 \quad 60 \%$ Die entsprechenden Nachweise sind gegenüber der obersten Abfallbehörde des jeweiligen Bundeslands zu führen.

Werden diese Anforderungen nicht erfüllt, so wird die Genehmigung für duale Erfassungs- und Verwertungssysteme nicht erteilt. Gegebenenfalls wird eine bereits erteilte Genehmigung widerrufen. Im Falle des Widerrufs greift nach einer Übergangsfrist von sechs Monaten wieder die allgemeine Rücknahme- und Verwertungspflicht von Herstellern und Vertreibern.

Quelle: BMU-Mitteilung vom 21. 09. 1992 